\title{
Interference of Robust Tori on the Resonance Overlap
}

\author{
R. Egydio de Carvalho, ${ }^{*}$ Caroline G.L. Martins, and G.M. Favaro ${ }^{\dagger}$ \\ Departamento de Estatística, Matemática Aplicada e Computação, \\ Instituto de Geociências e Ciências Exatas, Universidade Estadual Paulista, \\ UNESP, Av. 24A , 1515 - Bela Vista, 13506-900 Rio Claro, SP, Brazil
}

(Received on 30 June, 2008)

\begin{abstract}
We study a dynamical system which describes the overlap of resonances in a global integrable context and we present a new topological scenario for the reconnection of three resonance island chains in the presence of two robust tori. These tori induce this new scenario and they play the role of transport barriers which are relevant for plasma confinement in Tokamaks.
\end{abstract}

Keywords: Isochronous resonances; Resonance Overlap; Robust tori; Reconnection

\section{INTRODUCTION}

Isochronous resonances [1] constitute the most appropriate prototype to investigate the overlap of resonances. In generic dynamical systems the resonances come associated with chaos and when we say in overlap, we are usually saying in interactions of resonances immerse in a chaotic sea. The resonances are identified, in the phase space of the systems, through the visualization of separatrices which delimitate libration motion from the one of rotation. The separatrices are also denoted by islands and when they are far from each other, their neighborhoods are similar to the pendulum dynamics. As some control parameter is varied, the separatrices split and each resonance experiences the presence of the other and the resonance overlap occurs. This interaction, sometimes is also called by reconnection [2], occurs when the splitting of the separatrices are sufficiently close in such way that there are not any tori in the region of interaction between the separatrices. This scenario is well reported by Chirikov [3] but it does not allow us to visualize and understand the details of the overlap of resonances. In a complementary context, it was developed an integrable Hamiltonian scenario, with resonances of same order, where the overlap can occur without any interference of chaos [1]. The necessary condition to occur this configuration is to introduce a non-twist term in the non-perturbed system in order to allow the occurrence of, at least, two resonances but without breaking down the integrability of the system. These are the isochronous resonances. The non-twist condition for Hamiltonian flux [4,5] is naturally manifested in the rotation number or in the safety factor in the context of plasma physics [6]. The safety factor, in this case, presents a nonmonotonic profile which can trigger multiple instability modes [7]. For discrete systems, there are also some correlated studies on non-twist maps, associated with the idea of the isochronous resonances [8-11]. But the resulting topological configuration after the resonances overlapping can vary depending on the quantity of resonances which participate on the reconnection process, even though some patterns frequently appear. Besides the quantity of resonances, we introduce another agent which can interfere in the topological

${ }^{*}$ Electronic address: regydioerc.unesp.br

$\dagger$ Electronic address: gmfavaro@gmail.com rearrangement, it is the called robust torus [12]. This kind of torus survives intact to the effects of generic perturbations and it constitutes an efficient transport barrier [13]. In this sense we would like to present in this work a new scenario for the overlap of resonances when three isochronous resonances and two robust tori interact and participate in this process. The paper is organized as follows, in section 2 we present the strategy that we will follow to construct a Hamiltonian which foresees these dynamical constituents. The Hamiltonian is generated from the expansion of the Birkhoff-Gustavson normal form in the action-angle variables $[14,15]$. In section 3 , we particularize the obtained Hamiltonian for our purposes keeping only the relevant terms in this expansion. In section 4 we show the numerical results and finally in section 5 we present the final remarks and conclusions.

\section{REVIEWING THE RESONANT NORMAL FORM}

In order to introduce to the readers the Hamiltonian function which we have used to develop our studies, we will present a brief review of the formalism of the BirkhoffGustavson's Normal Form-BGNF around a stable fixed point for a generic autonomous system with two degree of freedom, which can be found in more details in [16]. The Hamiltonian is initially described in terms of the momentumposition variables $\left(p_{k}, q_{k}\right.$, for $k=1$ or 2 ).

The linear neighborhood of an elliptic fixed point is well approximated by harmonic oscillators while the nonlinearities are all included in a generic sum containing terms from third order in the $\left(p_{k}, q_{k}\right)$ variables. We start from the following Hamiltonian governing the dynamics of a particle,

$$
\begin{aligned}
H(\vec{q}, \vec{p}) & =\omega_{1}\left(\frac{p_{1}^{2}+q_{1}^{2}}{2}\right)+\omega_{2}\left(\frac{p_{2}^{2}+q_{2}^{2}}{2}\right)+ \\
& +\sum_{\vec{\alpha}+\vec{\beta}=3}^{\infty} k_{\vec{\alpha}, \vec{\beta}} q_{1}^{\alpha_{1}} \cdot q_{2}^{\alpha_{2}} \cdot p_{1}^{\beta_{1}} \cdot p_{2}^{\beta_{2}}
\end{aligned}
$$

where $k_{\vec{\alpha}, \vec{\beta}}$ is a parameter which can depend on $\alpha$ and $\beta$. We say that a resonance exists in the origin of the 4-d phase space if there is a commensurability condition between the frequencies of the type $\frac{\omega_{1}}{\omega_{2}}=\frac{s}{r}$, where $\frac{s}{r}$ is a rational number.

We perform a canonical transformation (CT) changing the 
variables $(\vec{p}, \vec{q})$ to the complex ones $\left(\vec{a}, \overrightarrow{a^{*}}\right)$ through,

$$
\vec{a}=\frac{1}{\sqrt{2}}(\vec{q}+i \vec{p}), \overrightarrow{a^{*}}=\frac{1}{\sqrt{2}}(\vec{q}-i \vec{p})
$$

or alternatively,

$$
\vec{q}=\frac{1}{\sqrt{2}}(\vec{a}+\overrightarrow{a *}), \vec{p}=\frac{i}{\sqrt{2}}(\overrightarrow{a *}-\vec{a})
$$

In this new set of variables the Hamiltonian is described by,

$$
\begin{aligned}
H(\vec{a}, \overrightarrow{a *}) & =\omega_{1}\left(a_{1} \cdot a_{1}^{*}\right)+\omega_{2}\left(a_{2} \cdot a_{2}^{*}\right)+ \\
& +\sum_{\vec{\alpha}+\vec{\beta}=3}^{\infty} k_{\vec{\alpha}, \vec{\beta}} a_{1}^{\alpha_{1}} \cdot a_{2}^{\alpha_{2}} \cdot a_{1}^{* \beta_{1}} \cdot a_{2}^{* \beta_{2}}
\end{aligned}
$$

According to Birkhoff's theorem [14] it is possible, in general, to eliminate high order terms from the series given above through successive time-independent $\mathrm{CT}$, in such way we get the expression,

$$
\begin{aligned}
H(\vec{a}, \overrightarrow{a *}) & =\left\{\begin{array}{l}
\omega_{1}\left(a_{1} \cdot a_{1} *\right)+\omega_{2}\left(a_{2} \cdot a_{2} *\right)+b_{4,0}\left(a_{1} \cdot a_{1} *\right)^{2}+b_{2,2}\left(a_{1} \cdot a_{1} *\right)\left(a_{2} \cdot a_{2} *\right)+\ldots \ldots \\
\ldots .+b_{0, N-1}\left(a_{2} \cdot a_{2} *\right)^{\frac{(N-1)}{2}}
\end{array}\right\} \\
& +\sum_{\vec{\alpha}+\vec{\beta}=N}^{\infty} k_{\vec{\alpha}, \vec{\beta}} \cdot a_{1}^{\alpha_{1}} \cdot a_{2}^{\alpha_{2}} \cdot a_{1}^{* \beta_{1}} \cdot a_{2}^{* \beta_{2}}
\end{aligned}
$$

where the coefficients $b_{i, j}$ represent the dependence of the frequencies on the oscillation amplitudes and the expression in curly brackets is of finite order and totally integrable. In general we are not able to extend this order to infinite but only to a finite order what means to make an approximation to the real motion, by neglecting terms in the summation. These terms, collectively, may be considered as perturbations since they are not reducible to an integrable form. But this truncating gives a good approximation to describe the motion near a stable equilibrium point. This truncate Hamiltonian is called Birkhoff's Normal Form (BNF).

A formal way to try to extend the BNF up the order $\left(\vec{a} \cdot \overrightarrow{a^{*}}\right)^{N}$ is to apply a CT $\left(\vec{a}, \overrightarrow{a^{*}}\right) \rightarrow\left(\vec{Z}, \overrightarrow{Z^{*}}\right)$ using the generating function,

$$
F=Z_{1} \cdot a_{1}^{*}+Z_{2} \cdot a_{2}^{*}+\sum_{\vec{\alpha}+\vec{\beta}=N}^{\infty} S_{\vec{\alpha}, \vec{\beta}} \cdot Z_{1}^{\alpha_{1}} \cdot a_{1}^{* \beta_{1}} \cdot Z_{2}^{\alpha_{2}} \cdot a_{2}^{* \beta_{2}}
$$

From this expression we immediately obtain the series for $a_{1}, a_{2}, Z_{1}^{*}, Z_{2}^{*}$. Reversing these last two series we get $a_{1}^{*}, a_{2}^{*}$ and substituting these results in the two first series we obtain $\left(\vec{a}, \overrightarrow{a^{*}}\right)$ explicitly in terms of $\left(\vec{Z}, \overrightarrow{Z^{*}}\right)$.

Only the quadratic term $\omega_{1}\left(a_{1} \cdot a_{1} *\right)+\omega_{2}\left(a_{2} \cdot a_{2} *\right)$, in eq. (5), gives a contribution of order $\mathrm{N}$ beside the linear approximation $\left(\vec{a}=\vec{Z} \quad, \quad \overrightarrow{a^{*}}=\overrightarrow{Z^{*}}\right)$. In all other terms we have to use this linear approximation to keep the series starting from N. After some algebraic manipulations we rewrite the Hamiltonian given in eq. (5) by,

$$
\begin{aligned}
H(\vec{a}, \overrightarrow{a *}) & =\left\{\begin{array}{l}
\omega_{1}\left(a_{1} \cdot a_{1} *\right)+\omega_{2}\left(a_{2} \cdot a_{2} *\right)+b_{4,0}\left(a_{1} \cdot a_{1} *\right)^{2}+b_{2,2}\left(a_{1} \cdot a_{1} *\right)\left(a_{2} \cdot a_{2} *\right)+\ldots . . \\
\ldots .+b_{0, N-1}\left(a_{2} \cdot a_{2} *\right)^{\frac{(N-1)}{2}}
\end{array}\right\} \\
& +\sum_{\vec{\alpha}+\vec{\beta}=N}^{\infty}\left\{k_{\vec{\alpha}, \vec{\beta}}-\left[\omega_{1}\left(\beta_{1}-\alpha_{1}\right)+\omega_{2}\left(\beta_{2}-\alpha_{2}\right)\right] S_{\vec{\alpha}, \vec{\beta}}\right\} a_{1}^{\alpha_{1}} \cdot a_{2}^{\alpha_{2}} \cdot a_{1}^{* \beta_{1}} \cdot a_{2}^{* \beta_{2}}
\end{aligned}
$$

Looking at the equation (7) we see that the case $\alpha_{1}=\beta_{1}$ and $\alpha_{2}=\beta_{2}$ gives a trivial contribution to the BNF, so a natural choice for $S_{\vec{\alpha}, \vec{\beta}}$ is given by,

$$
S_{\vec{\alpha}, \vec{\beta}}=\frac{k_{\vec{\alpha}, \vec{\beta}}}{\omega_{1}\left(\beta_{1}-\alpha_{1}\right)+\omega_{2}\left(\beta_{2}-\alpha_{2}\right)}
$$

When the frequencies ratio is irrational and $\alpha_{1} \neq \beta_{1}$ and $\alpha_{2} \neq \beta_{2}$, the expression (8) eliminates all terms in the summation of equation (7) and the BNF can be obtained for any order representing a global integrable system. On the other hand if the frequencies ratio is rational, the expression (8) diverges defining a resonance. So, exist many terms hidden in the summation of equation (7) which can not be elimi- 
nated and in this case the equation (8) is called BirkhoofGustavson's Normal Form (BGNF), or simply Resonant Normal Form.

The construction of the desired Hamiltonian which includes resonance structures and other invariant tori in a global integrable context imposes the choice of a single term, or a pair of conjugate complex terms. So, one way to choose an appropriate resonant term is when the sum $(\vec{\alpha}+\vec{\beta})$ is minimum, this is, based on the degree of the BNF we take one resonant term of superior degree. Therefore we $\operatorname{put}(\vec{\alpha}+\vec{\beta})=(r+s)$. Then eq. (7) is giving by,

$$
\begin{aligned}
H(\vec{a}, \overrightarrow{a *}) & =\left\{\begin{array}{l}
\omega_{1}\left(a_{1} \cdot a_{1} *\right)+\omega_{2}\left(a_{2} \cdot a_{2} *\right)+b_{4,0}\left(a_{1} \cdot a_{1} *\right)^{2}+b_{2,2}\left(a_{1} \cdot a_{1} *\right)\left(a_{2} \cdot a_{2} *\right)+\ldots \ldots \\
\ldots .+b_{0, N-1}\left(a_{2} \cdot a_{2} *\right)^{\frac{(N-1)}{2}}
\end{array}\right\} \\
+ & \left(a_{1}^{r} \cdot a_{2}^{* s}+a_{1}^{* r} \cdot a_{2}^{s}\right)+\text { HOT }
\end{aligned}
$$

where HOT means higher order terms, from $(r+s+1)^{t h}$ order. The equation (9) is a particular case of the BGNF.

The dynamics can be easily described in terms of the action-angle variables $(\vec{I}, \vec{\varphi})$ (we point out that $\varphi$ is a scalar, the arrow represents the two variables $\left.\varphi_{1}, \varphi_{2}\right)$ which are given by the transformation,

$$
\vec{a}=\sqrt{\vec{I}} \exp \left(i \cdot \overrightarrow{\varphi)} \quad, \quad \overrightarrow{a^{*}}=\sqrt{\vec{I}} \exp (-i \cdot \vec{\varphi})\right.
$$

in such way that,

$$
\vec{a} \cdot \overrightarrow{a^{*}}=\vec{I}=\frac{\overrightarrow{p^{2}}+\overrightarrow{q^{2}}}{2}
$$

consequently the momenta and positions are related with $(\vec{I}, \vec{\varphi})$ by

$$
\vec{q}=\sqrt{2 \vec{I}} \cos (\varphi) \quad, \quad \vec{p}=\sqrt{2 \vec{I}} \sin (\varphi)
$$

and the Hamiltonian, eq. (9), becomes,

$$
\begin{aligned}
H(\vec{I}, \vec{\varphi}) & =\omega_{1} I_{1}+\omega_{2} I_{2}+b_{4,0} I_{1}^{2}+b_{6,0} I_{1}^{3} \\
& +\ldots \ldots+b_{0, N-1} I_{2}^{(N-1) / 2}+ \\
& +\alpha\left(I_{1}^{r} I_{2}^{s}\right)^{1 / 2} \cos \left(r \varphi_{1}-s \varphi_{2}\right)
\end{aligned}
$$

where $\alpha$ is a control parameter or the perturbation parameter which was introduced only now to do not cause confusion. The effect of the added term is to generate a resonance of order $\mathrm{s}: \mathrm{r}$.

Finally, we make the linear $\mathrm{CT}(\vec{I}, \vec{\varphi}) \rightarrow(\vec{J}, \vec{\theta})$,

$$
\begin{array}{lc}
I_{1}=r J_{1} & I_{2}=J_{2}-s J_{1} \\
\theta_{1}=r \varphi_{1}-s \varphi_{2} & \theta_{2}=\varphi_{2}
\end{array}
$$

whose effect is to focus a single island of the resonance chain.

If we choose $\frac{\omega_{1}}{\omega_{2}}=\left(\frac{s}{r}\right)-\lambda$ with $\lambda$ a small constant we displace the resonance from the origin to a resonant torus. Initially we have put $\lambda=0$ in order to identify and add the resonant terms in eq. (9) and eq. (13), now we study the simplest approximation which is valid near $\lambda=0$, making $\lambda \neq 0$. The Hamiltonian, eq. (13), reads as,

$$
\begin{aligned}
& H(\vec{J}, \vec{\theta})= \\
= & \left\{\omega_{2}\left(J_{2}-r \lambda J_{1}\right)+b_{4,0}\left(r J_{1}\right)^{2}+b_{6,0}\left(r J_{1}\right)^{3}+\ldots \ldots . . .\right\}+ \\
+ & \alpha\left(r J_{1}\right)^{r / 2} .\left(J_{2}-s J_{1}\right)^{s / 2} \cos \left(\theta_{1}\right)
\end{aligned}
$$

Since $H(\vec{J}, \vec{\theta})$ does not depend on $\theta_{2}$ then its canonical conjugate variable $J_{2}$ is a global constant of motion, so the system is integrable because it has two degrees of freedom and the total energy and $J_{2}$ are constants. The nonintegrability and consequently the chaotic orbits will appear only when another perturbation depending explicitly on $\theta_{2}$ is turned on.

From the Hamilton equations of motion,

$$
\dot{\theta_{1}}=\frac{\partial H}{\partial J_{1}} \quad, \quad \dot{J_{1}}=-\frac{\partial H}{\partial \theta_{1}} \quad, \quad \dot{\theta_{2}}=\frac{\partial H}{\partial J_{2}} \quad, \quad \dot{J_{2}}=-\frac{\partial H}{\partial \theta_{2}}
$$

we can study the dynamics in the phase space $\left(J_{1}, \theta_{1}\right)$ by integrating numerically these differential equations and using the technique of the Poincare's section. The term in curly brackets in equation (15), the BNF, is also called non-perturbed Hamiltonian $H_{0}$ and from equation (16) it is defined the resonance condition as,

$$
\stackrel{\bullet}{\theta_{1,0}}=\frac{\partial H_{0}}{\partial J_{1}}=0
$$

This equation shows us where will occur the resonance due to the introduction of the resonant term. If $H_{0}$ is at least a cubic polynomial, then equation (17) results also in a polynomial whose lower order is two, what means that a single resonant term in the BGNF introduces more than one resonance chain in the phase space. These resonances are called isochronous resonances because all of them have the same order s : r. This is a very important point because we can theoretically introduces as much as resonance chains that we desire to develop the study. Since the distances among these resonances are controlled by the resonance condition, equation (17), and the widths of the separatrices are controlled by the perturbation parameter, $\alpha$ in equation (15), these resonances can interact and to overlap themselves. This process is called overlap, or reconnection, of integrable resonances. 
On the other hand if we introduce other terms in the perturbation of equation (15), all only $\theta_{1}$-dependent, they can be grouped in such way that a common pre-factor can be put in evidence playing a very interesting role in the dynamics. For instance, if this pre-factor is a polynomial with real roots, it means that the perturbation will be algebraically zero in these roots even when it is turned on. This gives origin to tori which survive intact to the effects of the perturbations in some particular regions of the phase space. These tori are called Robust Tori.

Due to this versatility and to the many possible configurations of Hamiltonians, the Birkhoff-Gustavson Normal Form is interpreted as a toy model.

\section{THE HAMILTONIAN DYNAMICS WITH ROBUST TORI AND ISOCHRONOUS RESONANCES}

Since the essential points for obtaining the Hamiltonian of a particular system are presented we will use this mechanism in order to generate the Hamiltonian of our study, which will present three resonance chains, two robust tori and a new scenario of overlap of resonances, generated by three $\theta_{1}$-dependent perturbations.

We will adapt the Hamiltonians given in equation (9), (13) and (15) for our purposes by adjusting the coefficients and the quantity of terms. So, equation (9) becomes,

$$
\begin{aligned}
H & =\left\{\left(a_{2} \cdot a_{2}^{*}+\frac{a_{1} \cdot a_{1}^{*}}{4}\right)-\frac{a}{2}\left(a_{1} \cdot a_{1}^{*}-c\right)^{2}+\frac{1}{4}\left(a_{1} \cdot a_{1}^{*}-c\right)^{4}\right\}+ \\
& +\alpha\left\{\left(b c^{2}-a\right)\left(a_{1}^{4} a_{2}^{*}+a_{1}^{* 4} a_{2}\right)-(2 b c)\left(a_{1}^{5} a_{1}^{*} a_{2}^{*}+a_{1}^{* 5} a_{1} a_{2}\right)+b\left(a_{1}^{6} a_{1}^{* 2} a_{2}^{*}+a_{1}^{* 6} a_{1}^{2} a_{2}\right)\right\}
\end{aligned}
$$

where $a, b, c$ and $\alpha$ are adjustable parameters. From the $\mathrm{CT}$ presented in equation (10) the Hamiltonian above is de-

scribed by,

$$
H=\left(I_{2}+\frac{I_{1}}{4}\right)-\frac{a}{2}\left(I_{1}-c\right)^{2}+\frac{1}{4}\left(I_{1}-c\right)^{4}+\alpha I_{2}^{1 / 2} \cos \left(4 \varphi_{1}-\varphi_{2}\right) I_{1}^{2}\left\{\left[c^{2} b-a\right]-2 c b I_{1}+b I_{1}^{2}\right\}
$$

or simply,

$$
\begin{aligned}
H & =\left(I_{2}+\frac{I_{1}}{4}\right)-\frac{a}{2}\left(I_{1}-c\right)^{2}+\frac{1}{4}\left(I_{1}-c\right)^{4}+ \\
& +\alpha I_{1}^{2} I_{2}^{1 / 2} \cos \left(4 \varphi_{1}-\varphi_{2}\right)\left[b\left(I_{1}-c\right)^{2}-a\right]
\end{aligned}
$$

The CT given in equation (14) produces then the following Hamiltonian,

$$
\begin{aligned}
H & =\left\{J_{2}-\frac{a}{2}\left(4 J_{1}-c\right)^{2}+\frac{1}{4}\left(4 J_{1}-c\right)^{4}\right\}+ \\
& +\alpha\left\{\left[b\left(4 J_{1}-c\right)^{2}-a\right]\left(4 J_{1}\right)^{2}\left(J_{2}-J_{1}\right)^{1 / 2} \cos \left(\theta_{1}\right)\right\}
\end{aligned}
$$

According with the discussion of the previous section, the resonance condition uses the unperturbed Hamiltonian given in the first curly bracket, of equation (21), so that three isochronous resonance chains can appear when a periodic $\theta_{1}$ perturbation is turned on. The positions of these resonance chains are labeled by $\left(J_{1}\right)_{+},\left(J_{1}\right)_{m}$ and $\left(J_{1}\right)_{-}$. Then the localizations of the three resonance chains are,

$$
\left(J_{1}\right)_{+}=\frac{c+\sqrt{a}}{4} \quad, \quad\left(J_{1}\right)_{m}=\frac{c}{4} \quad, \quad\left(J_{1}\right)_{-}=\frac{c-\sqrt{a}}{4}
$$

In the second curly bracket there is the desired perturbation with a pre-factor, in the square bracket, which is a quadratic polynomial in the $J_{1}$ action. Depending on the values of $a, b, c$, the pre-factor will present two real roots, which we call $\left(J_{1}\right)_{R+}$ and $\left(J_{1}\right)_{R-}$, and over these values of $J_{1}$, the perturbations are algebraically null even for any nonnull value of $\alpha$. Hence, two robust tori will be present in the phase space in the following positions,

$$
\left(J_{1}\right)_{R+}=\frac{c+\sqrt{a / b}}{4} \quad, \quad\left(J_{1}\right)_{R-}=\frac{c-\sqrt{a / b}}{4}
$$

We observe from these equations that the parameter $b$ changes the position of the robust tori, while the positions of the resonances are not affected by it. This characteristic makes the parameter $b$ as a relevant one in order to realize the interaction between the robust tori with the resonance structures.

Since the system is globally integrable, because $\theta_{2}$ is a cyclic variable, $J_{2}$ may play the role of a reduced Hamiltonian $J_{2}=J_{2}\left(J_{1}, \theta_{1}\right.$, Energy $=$ constant $)$ with $\theta_{2}$ doing the role of canonical time. In this case the equations of motion read as, 


$$
\dot{J}_{1}=-\left(\frac{\partial H / \partial \theta_{1}}{\partial H / \partial J_{2}}\right) \quad, \quad \dot{\theta_{1}}=\left(\frac{\partial H / \partial J_{1}}{\partial H / \partial J_{2}}\right) \quad, \quad \dot{J}_{2}=\left(\frac{\partial H / \partial \theta_{2}}{\partial H / \partial J_{2}}\right) \quad, \quad \dot{\theta}_{2}=\left(\frac{\partial H / \partial J_{2}}{\partial H / \partial J_{2}}\right)=1
$$

The phase space of this integrable system can be exploited through the numerical integration of this set of equations. Using the reduced Hamiltonian, each initial condition on the phase space corresponds to a torus with a specific $J_{2}$ and a global constant energy. We point out that to break down the integrability of the system it is sufficient to introduce any perturbation which depend explicitly on $\theta_{2}$. In the next section we present the numerical calculations for different geometrical configurations.

\section{RECONNECTION INVOLVING INTEGRABLE RESONANCES}

In order to investigate the effect of the robust tori on the overlap of the resonances, we have fixed the set of parameters of the Hamiltonian presented in equation (21) and we let $\alpha$ and $b$ as adjustable parameters for developing our study. We have chosen $a=4.0, c=16.0$, Enenrgy $=100.0$ and three values for $b$ to investigate the dynamics, $b=0.17$ which corresponds to two external robust tori and far from the three resonance chains, $b=0.70$ where the robust tori continue external but now very close to the resonance chains and $b=2.0$ where the robust tori are intercalated between the resonance chains. The parameter $\alpha$ controls the amplitude of the perturbations what implies in increasing or decreasing the widths of the separatrices, or the islands, in such way that the separatrix of a chain can attain another one of other chain and start the overlap of resonances. We emphasize that this kind of overlap of integrable resonances does not create any chaotic layer in the neighborhood of a separatrix, as described by Chirikov, but a topological rearrangement involving invariant tori will emerge. This reconnection process in the presence of robust tori is new and different of what is usually discussed in the literature. The separatrix can also interact with a robust torus, depending on the parameter $b$, and generate a very rich geometrical structure.

Case $b=0.17$, the robust tori are external to the chains and far from them. The next four figures illustrate the dynamics,

For $\alpha=1.0 \cdot 10^{-4}$, figure $1 \mathrm{a}$, we observe three noninteracting resonance chains. The range of $\theta_{1}$ was amplified up $4 \pi$ to guide the eyes. For $\alpha=2.0 \cdot 10^{-4}$, figure $1 b$, the separatrices of the two superior chains are practically in contact and a global reconnection will start. For $\alpha=8.0 \cdot 10^{-4}$, figure $1 \mathrm{c}$, the three chains have already overlapped themselves, now the system presents a very interesting dynamical scenario. The manifold which emanated from the hyperbolic fixed point of the upper separatrix now it does a loop involving the elliptic fixed point of the intermediate separatrix. On the other hand, the manifold which emanated from the intermediate separatrix now it does two loops, like an eight, involving the elliptic fixed points of both, the upper and the lower separatrices. This eight-like loop is contoured by the manifold which emanates from the lower separatrix.
This is the topological rearrangement after the reconnection of the three integrable resonances without the influence of the robust tori for moderate perturbation. For $\alpha=2.0 \cdot 10^{-3}$, figure $1 \mathrm{~d}$, an inverse supercritical pitchfork bifurcation has occurred involving the three fixed points of the eight-like loop, the two elliptic fixed points coalesce with the hyperbolic fixed point resulting in a single elliptic fixed point and libration tori. The final geometrical configuration in this case presents only two resonance island chains, while the third one has disappeared after the reconnection process and the cited bifurcation.

Case $b=0.70$, the robust tori are external to the chains but close from them. The next five figures illustrate the dynamics,

For $\alpha=2.0 \cdot 10^{-4}$, figure $2 \mathrm{a}$, the three chains are not interacting yet and the two robust tori are very close to the external separatrices. For $\alpha=4.0 \cdot 10^{-4}$, figure $2 b$, the amplitudes of the islands are sufficiently large for the occurrence of the reconnection process but with a quite different topology. It is visible that the robust tori limit the growing of the resonance islands, the librations near the robust tori are flattened. As the overlap process starts we see the stretching of the islands but the robust tori imposes a limitation to this stretching. The hyperbolic fixed point from the upper separatrix interacts with the robust torus and a subcritical pitchfork bifurcation occurs, generating two others hyperbolic and one elliptic fixed points, defining a small island. This elliptic fixed point will remain in $\theta_{1} \approx \pi$ forever while the two hyperbolic ones will 'walk' along the robust tori as the amplitude of the associated island increases. The manifolds of the hyperbolic fixed point of the intermediate chain again make an eight-like loop involving the elliptic fixed points of the external separatrices. The manifold which emanates from the inferior hyperbolic fixed point, initially, makes a loop involving the elliptic fixed point of the intermediate chain and it arrives at the hyperbolic fixed point which has born over the upper robust torus. In a next moment, for $\alpha=8.0 \cdot 10^{-4}$, figure $2 \mathrm{c}$, the hyperbolic fixed point of the lower separatrix also interacts with the robust torus and a similar bifurcation occurs over it. Another bifurcation has occurred eliminating the eight-like loop. For $\alpha=2.0 \cdot 10^{-3}$, figure $2 \mathrm{~d}$, we observe the final configuration with one more island chain, besides what there was in the initial configuration, the phase space now presents four island chains. The robust tori also play the role of separatrix in this configuration. This topological rearrangement corresponds to a new scenario for the overlap of resonances. In figure $2 \mathrm{e}$ we see the bifurcation diagram with the original hyperbolic fixed point identified by $H_{1}$ and the others, which have born after the bifurcation, identified by $E_{1}, H_{2}$ and $H_{3}$.

Case $b=2.0$, the robust tori are intercalated between the chains. The next five figures illustrate the dynamics,

In figure $3 \mathrm{a}$, for $\alpha=1.0 \cdot 10^{-4}$, the external resonance chains are very close of the robust tori but they are not interacting yet. For $\alpha=2.0 \cdot 10^{-4}$, figure $3 b$, we observe that 

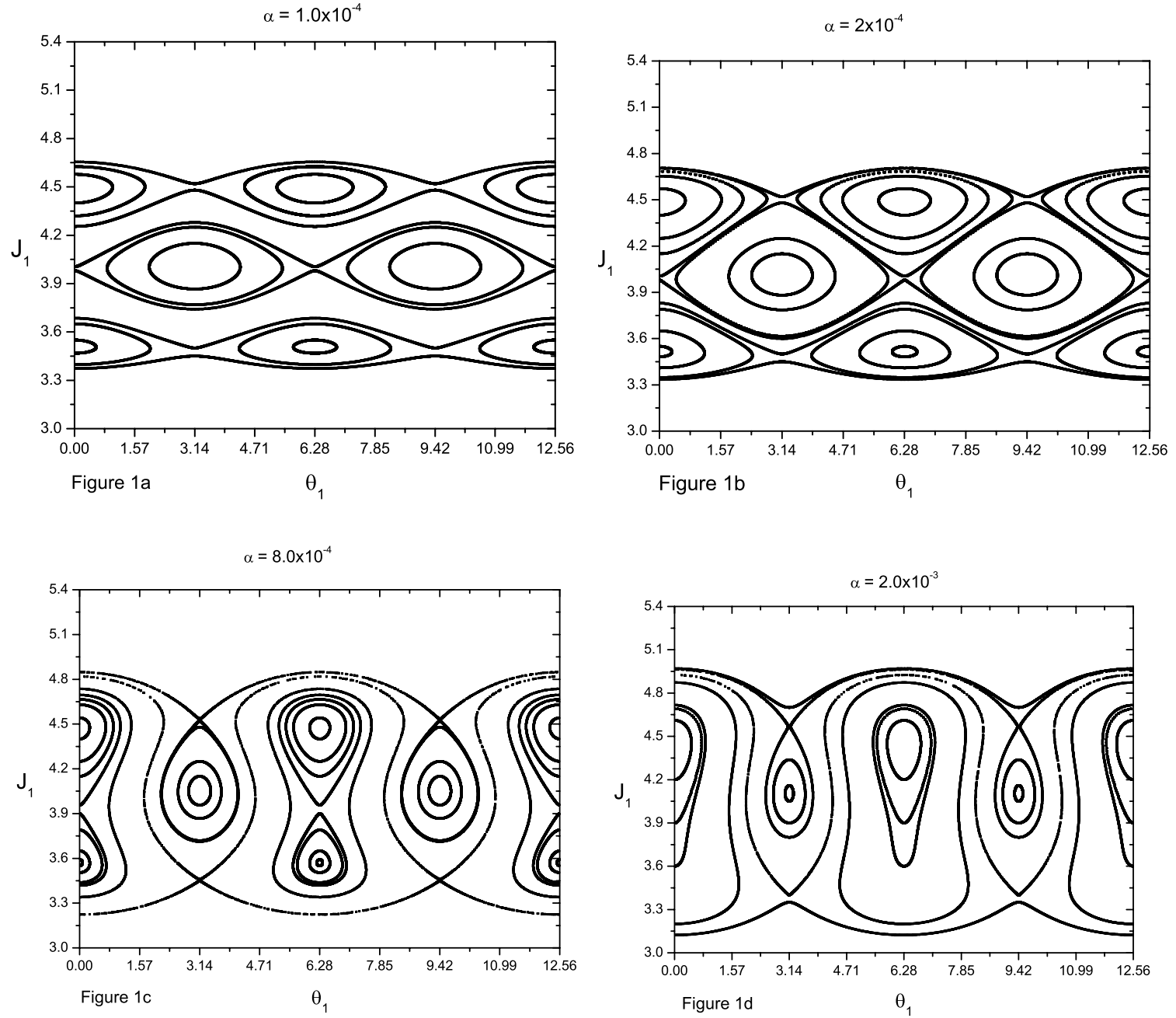

FIG. 1: Case $b=0.17$, without Robust Tori. (a) Three non-interacting isochronous resonances; (b) Three near interacting isochronous resonances; (c) Three isochronous resonances after reconnection; (d) Three isochronous resonances after reconnection and after a bifurcation.

the interaction between the separatrices with the robust tori has already started, the libration tori are also flattened due to the presence of the robust tori and a similar subcritical pitchfork bifurcation occurred from the contact of the hyperbolic fixed point, identified by $H_{1}$ in figure $3 \mathrm{a}$, with the superior robust torus. Two hyperbolic and one elliptic fixed points have emerged introducing a new small island below the upper robust torus. It is important to emphasize that from the beginning of the interaction between the island chains with some robust torus, this torus becomes also separatrices of the islands over it. For $\alpha=3.0 \cdot 10^{-4}$, figure 3c, other analogous bifurcation occurred over the lower robust torus and another island was introduced in the region of the intermediate resonance chain. Even though the amplitudes of the external separatrices have been stretched, the intermediate separatrix is completely limited by both robust tori. Still in the intermediate region, we see that both new islands are enlarged and each one is going towards the other. For $\alpha=4.0 \cdot 10^{-4}$, figure $3 \mathrm{~d}$, the elliptic fixed points of these new islands have bifurcated with the hyperbolic fixed point at $\theta_{1}=2 \pi$ through an inverse supercritical pitchfork bifurcation and the final con- figuration is the same as the one presented in figure $2 d$. Even though the figures $2 \mathrm{~d}$ and $3 \mathrm{~d}$ are totally similar, the processes for which these configurations have been obtained were different. In figure $3 \mathrm{e}$ we follow the behavior of the fixed points involved in the bifurcation process on the upper robust torus, where we observe that the elliptic one, $E_{1}$, remains at $\theta_{1}=2 \pi$ while the two hyperbolic $H_{2}$ and $H_{3}$ spread up a maximum limit. The presence of the robust tori during the reconnection of the islands also produces the new scenario for the overlap of resonances.

\section{FINAL REMARKS AND CONCLUSION}

In this work we have developed a Hamiltonian through the expansion of the Resonant Normal Form around a stable fixed point in order to study the overlap of integrable resonances. According to Chririkov's criterion, a resonance island chain can experience the presence of another resonance when the distance between them is of the same order than the sum of the semi-width of the corresponding separatri- 

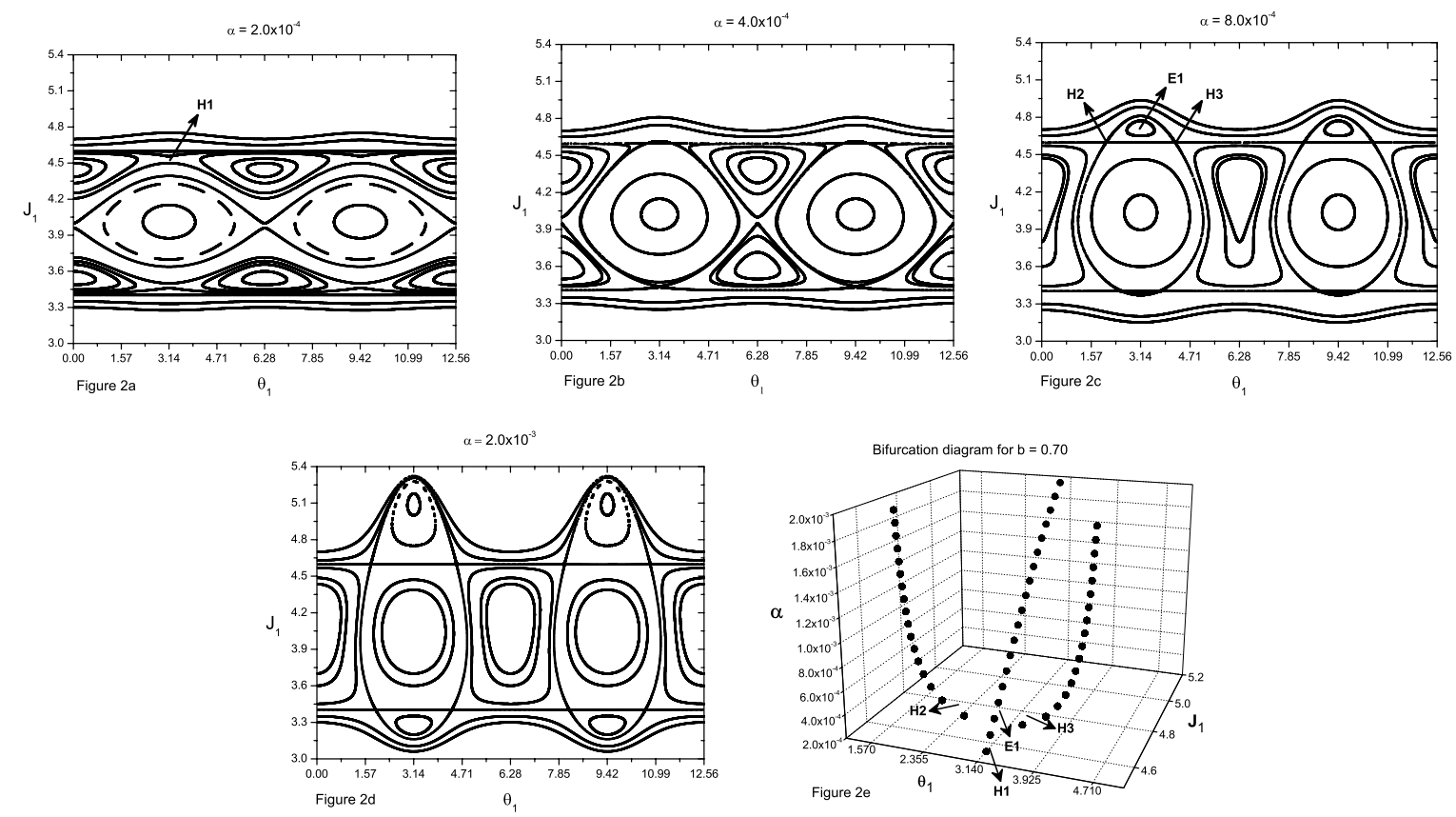

FIG. 2: Case $b=0.70$, with two robust tori. (a) three isochronous resonances; (b) three isochronous resonances interacting with both robust tori; (c) three isochronous resonances and a bifurcation at $\theta_{1}=2 \pi$; (d) it is a new topological configuration; (e) spatial representation for the bifurcation involving the fixed points identified in figure $2 \mathrm{c}$.

ces. But before the exact contact between the islands it occurs the splitting of the separatrix and the region between the near-islands is filled by homoclinic chaos. Hence the Chirikov's sense for the overlap of resonances is in a nonintegrable context and usually resonance is associated with chaos. Then the single way to study overlap of resonances in an integrable Hamiltonian context is to have a non-twist condition, or a non-monotonic rotation number, for the nonperturbed Hamiltonian and to turn on a periodic perturbation. This technique will produce naturally many, or at least two, isochronous resonance island chains in such way the overlapping process can be observed in a global integrable system. In our case in this work, we introduced three resonance chains controlled by the same perturbation parameter. We have chosen a set of three $\theta_{1}$-perturbations which have a common algebraic form with a quadratic pre-factor in the $J_{1}$ action. The real roots of this pre-factor define the values of the positions of the robust tori where the perturbation set is algebraically null. So, the system we have studied has three isochronous resonances, two robust tori, a non-monotonic rotation number and a periodic integrable perturbation. The obtained patterns for the overlap of resonances when the robust tori participate of the reconnection process are totally new for our knowledge. When the robust tori are distant from the resonances region the obtained pattern is not usual but it is expected in some sense. The relevance of this study for the field of non-linear dynamics comes from the richness of the dynamics of this toy model and certainly from the results obtained with the Hamiltonian developed above. But our interest is also to give a contribution to the field of plasma physics because this concept of robust tori can be adapted in the Hamiltonian approaches used to confine plasma in Toka- maks since the control of chaotic magnetic fields in Tokamaks is a very important question [17]. It is also well known that there is much instability inside the plasma chamber and many efforts are carried to prolong the time of plasma confinement. The integrable approach that we have presented here does not fit the actual chaotic scenario involving the lines of fields but it is possible to prepare an experimental set up which is well represented by a Hamiltonian with a vanishing perturbation on robust tori. Even that these robust tori do not survive intact to the effects of the several modes of perturbation, they certainly will constitute a powerful stickiness which will improve the plasma confinement.

The Hamiltonian function typically used in plasma confinement approaches, in Tokamaks, has the form [18, 20],

$$
H(J, \theta)=\left\{\frac{J^{2}}{2}-\alpha \frac{J^{3}}{3}\right\}+\kappa \cos \left(m_{0} \theta\right) \sum_{k=-\infty}^{\infty} \delta\left(t-\frac{2 \pi}{N_{r}} k\right)
$$

where the curly brackets corresponds to the unperturbed nontwist Hamiltonian foreseeing two isochronous island chains. The other term, corresponds to a periodic perturbation acting discretely in time (the sum of kicks) with a constant prefactor $\kappa$, which does not introduce any robust tori. Hence, having in mind this Hamiltonian and our proposal to investigate the effect of robust tori on the reconnection process we have done the following: i) we introduced one more island chain by increasing the order of the polynomial unperturbed Hamiltonian; ii) we treated the pre-factor $\kappa$ as a truncated series and we kept terms up the second order in the action $J_{1}$, what introduces two robust tori. For the purpose of plasma confinement it would be sufficient to take only the first order term. Finally, we consider the periodic perturbation as a continuous function. With these considerations and the 

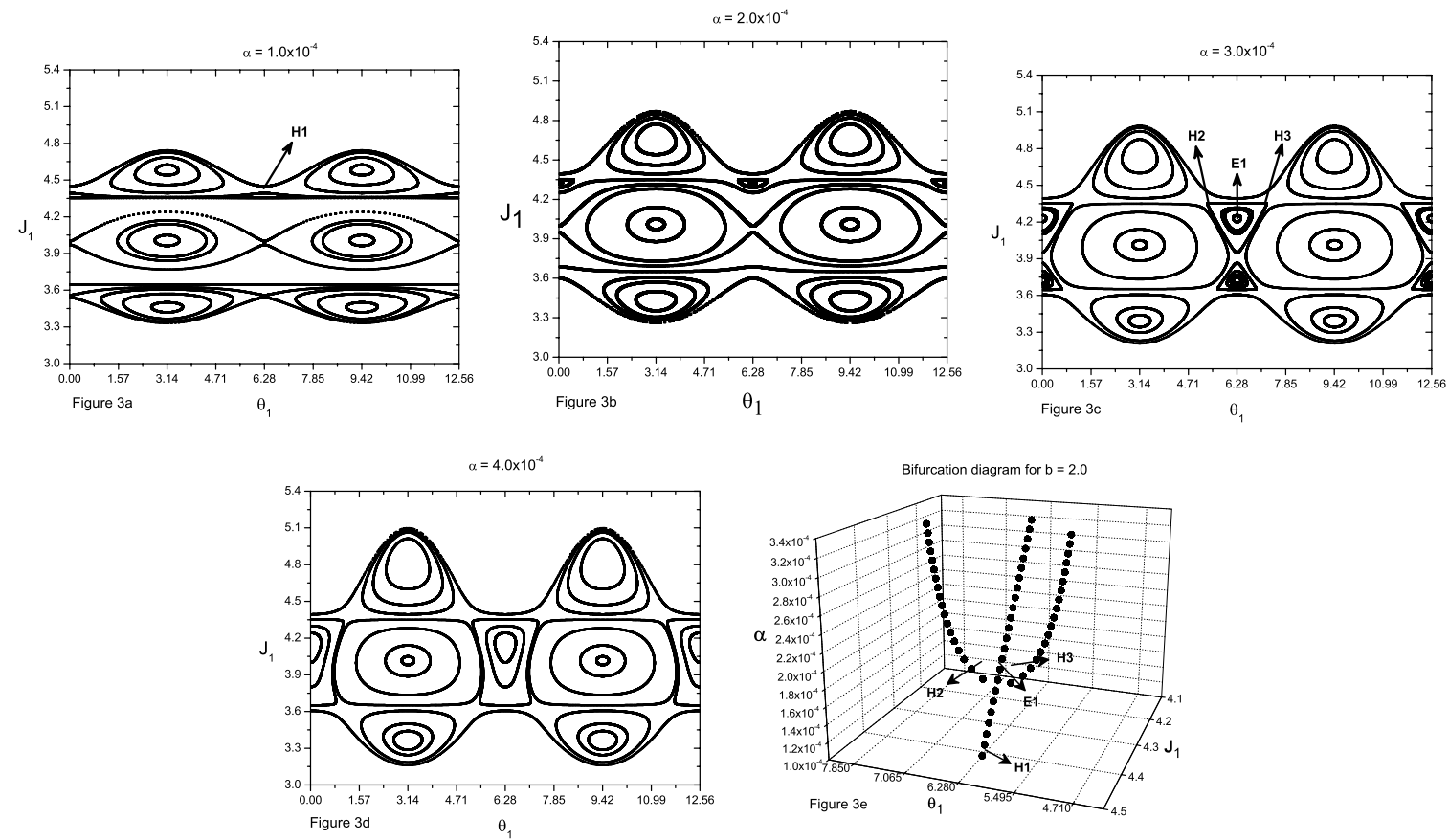

FIG. 3: Case $b=2.0$, with two intercalated robust tori. (a) three isochronous resonances; (b) three interacting isochronous resonances. The pattern of this interaction is different of the one observed in the set of figures 2 ; (c) a new reconnection pattern has occurred at $\theta_{1}=2 \pi$; (d) a bifurcation is observed for $\theta_{1}=2 \pi$; (e) spatial representation for the bifurcation involving the fixed points identified in figure $3 \mathrm{c}$.

technique of the BGNF we get the Hamiltonian expressed in equation (21), which is directly related with the typical Hamiltonian used by the plasma Physicists.

Besides this technique of robust tori, the resonance structures that we have presented for the reconnection process will allow an understanding a little better of how some structures are destroyed and how some stickiness can be generated inside the plasma chamber [18]. We also point out that even though there are non-twist maps which approach the plasma dynamics in Tokamaks $[19,20]$ they do not carry any robust tori. So, the inclusion of robust tori in the non-twist maps can improve the theoretical understanding of plasma confinement and simultaneously to suggest some new experi- mental setup [21]. This question will be present in a further work. As a final comment we would like to emphasize that the reconnection patterns obtained, from the resonant normal form, are quite general and this technique can be applied in any field of science.

\section{Acknowledgements}

We would like to thank the Brazilian scientific agency FAPESP for financial support through the processes numbers 06/51653-0 and 07/54372-5.
[1] R. Egydio de Carvalho and A.M. Ozório de Almeida, 1992 Phys. Lett. A 162457.

[2] E. Priest and T. Forbes, 2000 Magnetic Reconnection - MHD Theory and Applications (Cambridge University Press).

[3] B.V. Chirikov, 1979 Phys. Rep. 52265.

[4] G. Voyatzis and S. Ichtiaroglou 1999 Int. J. Bifurcation and Chaos 9(5) 849.

[5] G. Stagika and S. Ichtiaroglou, 2000 Cel. Mech. and Dyn. Astron. 78151.

[6] Wesson J 1987 Tokamaks (Oxford University Press).

[7] W. Kerner and H. Tasso, 1982 Phys. Rev. Lett. 49654.

[8] J.E. Howard and Hohs S.M. 1984 Phys. Rev. A 29418.

[9] J.P. van der Wheele, T.P. Valkering, H.W. Capel and T. Post, 1988 Physica A 153283.

[10] J.P. van der Wheele and T.P. Valkering, 1990 Physica A 169 42.
[11] J.E. Howards and J. Humpherys, 1995 Physica D 80256.

[12] R. Egydio de Carvalho and G.M. Favaro, 2005 Physica A 350 173.

[13] M. Roberto, E.C. da Silva, I.L. Caldas and R.L. Viana, 2004 Physica A 342363.

[14] F.G. Gustavson, 1966 Astron. J. 71670.

[15] A.M. Ozório de Almeida, 1990 Hamiltonian Systems: Chaos and Quantization (Cambridge University Press).

[16] R. Egydio de Carvalho, 1993 Nonlinearity 6973.

[17] I.L. Caldas, R.L. Viana, M.S.T. Araújo, A. Vannucci, E.C. da Silva, K. Ullmann and M.V.A.P. Heller, 2002 Braz. J. Phys. 32(4) 980.

[18] M. Roberto, E.C. da Silva, I.L. Caldas and R.L. Viana, 2004 Physica A 342363.

[19] J.S.E. Portela, I.L. Caldas and R.L. Viana, 2008 Eur. Phys. J. Special Topics 165195. 
[20] M. Roberto, E.C. da Silva, I.L. Caldas and R.L. Viana R L 2004 Braz. J. Phys. 34(4B) 1759.

[21] M. Dreval, C. Xiao, D. Trembach, A. Hirose, S. Elgriw, A.
Pant, D. Rohraff and T. Niu, 2008 Plasma Phys. Control. Fusion 50095014. 